\title{
NONDISCOGENIC SCIATICA SYNDROME, A REPORT ON 41 PATIENTS.
}

\section{Thamer Ahmed Hamdan * , Daren F. Lui ${ }^{\#}$ \& Raed Jasim Chasib ${ }^{\circledR}$}

* MB,ChB, FRCS, FICS, FACS, FRCP, American Board of Neurological \& Orthopaedic Surgery, Professor of Orthopedic Surgery, Research Fellow in Saint George's University Hospital, London, Visiting Professor, Imperial College, London, UK. \# MCh, FRCS Trauma \& Orthopaedics, Consultant Trauma, Orthopaedic, and Spine Surgeon, Saint George's University Hospital, London, UK. ${ }^{\circledR}$ MB,ChB, DOS, FICMS, FABMS, Basrah Teaching Hospital- Iraq, Department of Surgery, Orthopaedic Unit.

\begin{abstract}
41 patients with clinical features of discogenic sciatica during the last 25 years, proved later on by investigations and imaging to be a nondiscogenic in origin, The aim of the study is to inform spinal surgeons of possible differential diseases that mimic discogenic sciatica. All patients present clinically with a sciatica. They were recorded and treated accordingly and sent for investigations. Patients proved to have discogenic sciatica were excluded. Others further investigated and followed until finding a cause for the symptoms and recorded as nondiscogenic sciatica syndrome. 22 individual aetiologies were found to cause nondiscogenic including infectious, traumatic, oncogenic, degenerative, metabolic and ischemic causes.

In conclusion a proper history and thorough physical examination, in addition to imaging and investigations were very useful in detecting the exact etiology of sciatica. The pain pattern and the related clinical features were the guide for the diagnosis. High index of suspicion is vital to achieve the definite diagnosis to avoid missing the diagnosis of nondiscogenic sciatica. One should maintain a high index of suspicion in patients with intractable sciatica.

Keywords: [Nondiscogenic Sciatica syndrome, pseudosciatica, spine surgery, radicular pain.]
\end{abstract}

\section{Introduction}

ciatica is a syndrome characterized by pain radiating from back to the buttock and lower extremity along its posterior or lateral aspect and it is frequently caused by lumbar disc herniation. The nondiscogenic sciatica in our series presented with the same clinical features, but the cause was not disc prolapse. Often intra spinal or extra spinal pathology may affect the sciatic nerve through the roots of the sciatic nerve or through its course from the sacral foramenas downwards. Almost all pathological processes may lead to nondiscogenic sciatica such as infective, inflammatory, traumatic, neoplastic or degenerative disease. Clinicians are usually familiar with discogenic causes of sciatica. The uncommon causes are usually missed because of the low index of suspicion and the positive diagnostic tools focus on the lumbar spine. What makes the diagnosis more difficult are the findings of false positive MRI findings which can be reported in asymptomatic patients. There can also be positive discogenic pathology masking another cause. Dudeney et al. ${ }^{1}$ Reported in $20 \%$ of sciatica is due to discogenic and nondiscogenic cause. We confirmed this finding in our report in this article with one case of a lady with dual pathology with a disc prolapse and tumor of the lumbar root. The diagnosis of sciatica cannot be decided on MRI findings alone and correlation with the clinical findings is always required. Spinal MRI scans may reveal a high rate of abnormal signs in asymptomatic individuals who do not suffer from back pain. 
Studies regarding the diagnostic specificity of Lumbar MRI have been insufficient. Examining the whole patient's body in a systematic method keeping in mind pathology that mimic radiculopathy should help to reduce the incidence of misdiagnosis. The incidence of lumbar radiculopathy in general is approximately $3 \%-5 \%{ }^{2}$. In $10 \%$ of sciatica it is related to nondiscogenic aetiology. The differentiation between discogenic and nondiscogenic sciatica can be challenging to the clinician because the course of the sciatic nerve passes through different anatomical structures, all of which may lead to radicular pain. Tenderness to deep infragluteal palpation, a positive Tinnels sign or motor deficits are findings of pathology occurring that may no equivocate a discogenic source in the lumbar spine. Therefore, one should consider scheduling patients for an MRI gluteal and lumbar regions in order to better elucidate nondiscogenic sciatica pathology ${ }^{3}$. Some medications like statins, antivirals and antifibrillation drugs may cause myogenic or neurogenic pain simulating sciatica ${ }^{4}$. It can be confusing to localize the pain generator in nondiscogenic sciatica so it is important to be able to characterize the events leading to the onset of symptoms, factors that alleviate and exacerbate the pain, the pain location, associated symptoms, and any relevant medical history like malignancy.
The objectives of this study are to present a series of patients with nondiscogenic sciatica and review the proper technique for diagnosing this hidden pathology. It presents an overview of the different causes of intra spinal and extra spinal causes of nondiscogenic pain.

\section{Patients and methods}

All patients present clinically with a sciatica recorded and sent them for investigations, these investigations include:

- Plain x- ray

- CT scan

- MRI scan

- Hematological and biochemistry tests.

- EMG and nerve conduction study

Any patient proved to have discogenic sciatica is excluded. The others underwent further investigation in order to find a cause for the symptoms. Those with pathological findings were recorded as nondiscogenic sciatica syndrome. Follow up of patients for up to two years.

A systematic review of literature published in English language utilizing PubMed, Medline and Ovid, searching for the keywords nondiscogenic sciatica, pseudosciatica and radicular pain.

\section{Results}

The total number of patients were 41 patients, males were $26(63.4 \%)$ and $15(36.6 \%)$ were female and their age range between 22- 65 years. The cases were collected over a 25 years period by the senior author and all presented with cardinal features of discogenic sciatica. All were initially diagnosed as suspected discogenic sciatica syndrome. They were admitted for further evaluation, investigation and imaging. Tests included blood tests, plain X- ray of the lumbosacral spine, chest, pelvis and femoral head, EMG and nerve conduction study, MRI of lumbar spine and pelvis, CT scan of lumbar spine and pelvis, special immunological and complex investigation according to the specified condition. The discovered pathologies after thorough investigations are tabulated (Table 1) and reveal 22 causes. The main aetiology includes thirteen cases $(31.7 \%)$ of malignant 
lesion, five cases of infections and infestations (12.2\%), nine cases of intrathecal tumors as well as synovial cyst of the facet joint $(22 \%)$.

Table 1: Causes of non discogenic sciatica in our study

\begin{tabular}{|c|c|c|c|}
\hline Sequence & Case & Number & Percentage (\%) \\
\hline 1 & $\begin{array}{c}\text { Secondaries in the sciatic nerve from } \\
\text { bronchogenic carcinoma }\end{array}$ & 1 & 2.4 \\
\hline 2 & $\begin{array}{l}\text { Undifferentiated malignant neural tissue tumor } \\
\text { in the gluteal region }\end{array}$ & 1 & 2.4 \\
\hline 3 & $\begin{array}{c}\text { Carcinomatous neuropathy due to } \\
\text { bronchogenic carcinoma }\end{array}$ & 2 & 4.9 \\
\hline 4 & Ewing sarcoma of the pelvis & 2 & 4.9 \\
\hline 5 & Solitary myeloma in the pelvis & 1 & 2.4 \\
\hline 6 & Leiomyosarcoma of the paraspinal muscle & 1 & 2.4 \\
\hline 7 & Spinal secondaries in the body of L4 vertebrae & 2 & 4.9 \\
\hline 8 & Retroperitoneal sarcoma & 3 & 7.3 \\
\hline 9 & Brucellosis of the spine & 2 & 4.9 \\
\hline 10 & Tuberculosis of sacroiliac joint & 1 & 2.4 \\
\hline 11 & Typhoid spine & 1 & 2.4 \\
\hline 12 & Hydatid cyst of L4 lumbar vertebra & 1 & 2.4 \\
\hline 13 & Intrathecal tumors & 7 & 17.1 \\
\hline 14 & Synovial cyst of the facet joint & 2 & 4.9 \\
\hline 15 & Schwannoma of the common peroneal nerve & 1 & 2.4 \\
\hline 16 & $\begin{array}{c}\text { L4- L5 disc prolapse and Schwannoma of L5 } \\
\text { nerve root }\end{array}$ & 1 & 2.4 \\
\hline 17 & Neuroma of the lumbar root L4-L5 & 1 & 2.4 \\
\hline 18 & Early osteoarthritis of the hip joint & 2 & 2.4 \\
\hline 19 & Avascular necrosis of the femoral head & 3 & 7.3 \\
\hline 20 & Piriformis syndrome & 2 & 4.9 \\
\hline 21 & Trauma to the gluteal region & 2 & 4.9 \\
\hline 22 & Painful diabetic neuropathy of the sciatic nerve & 2 & 4.9 \\
\hline
\end{tabular}


Five patients had positive lumbar MRI for disc prolapse (12.2\% had nondiscogenic sciatica and positive asymptomatic MRI finding of lumbar disc prolapse). A retroperitoneal sarcoma was discovered in two cases, one case of painful diabetic neuropathy, one case of carcinomatous neuropathy (bronchogenic carcinoma) and one case of avascular necrosis of the femoral head.

One significant case is a 35 years lady present with L4- L5 big sequestrated disc herniation and Schwannoma of L4 nerve root at the same site [Figure 1], because we can't determine which pathology cause her symptoms we consider it as a case of discogenic and nondiscogenic sciatica syndrome.
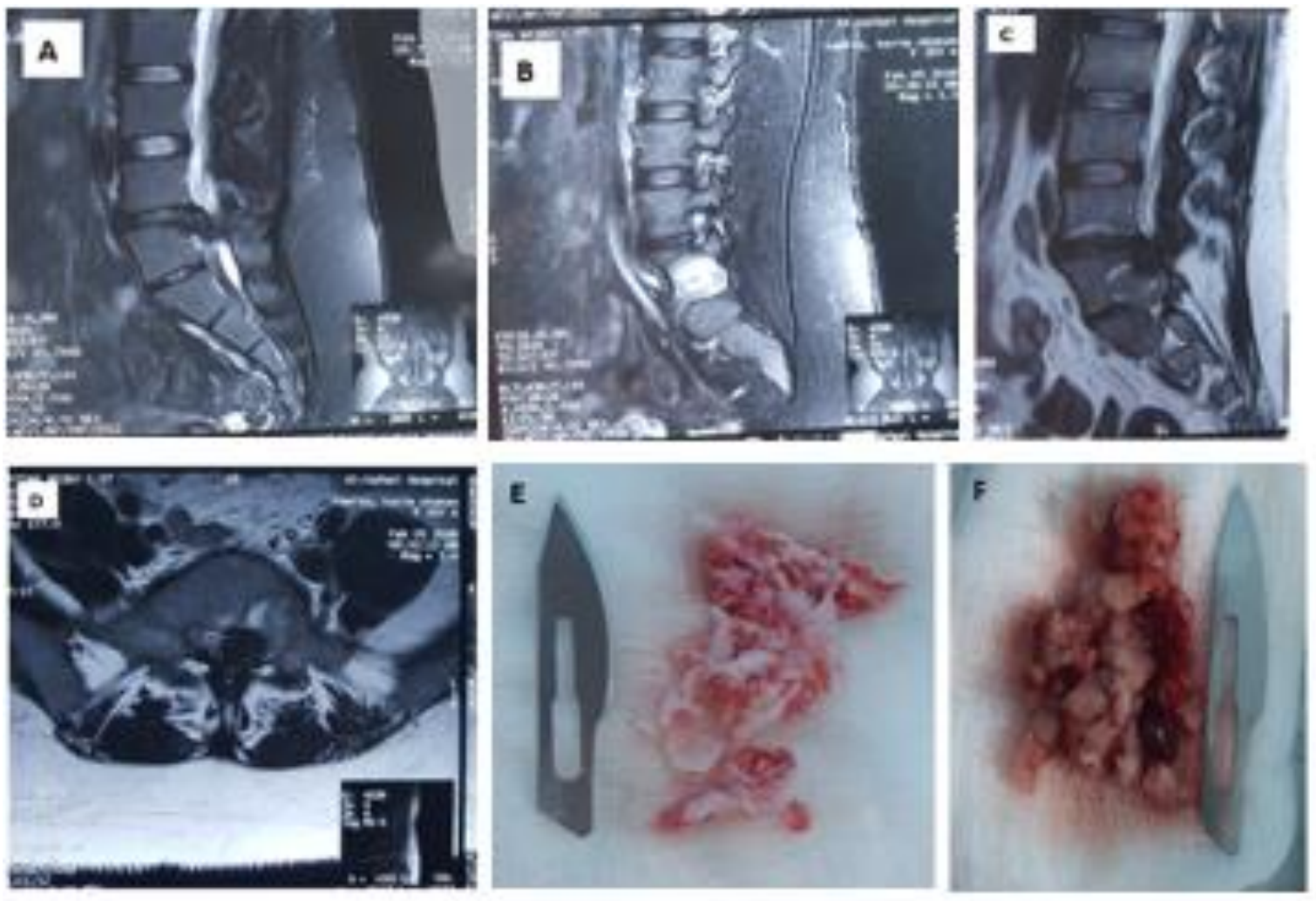

Figure 1: Discogenic and nondiscogenic sciatica in same site.( A)- This picture shows patient sagittal MRI section with sequestrated disc prolapse at L4-5,( B)- another sagittal section of same patient shows a big tumor at the level of L5 vertebral body, (C)sagittal section shows both disc prolapse and tumor at same site, (D)- axial MRI section shows disc material and tumor at same level, (E)- postoperative sample of disc material, (F)- postoperative tumor material

\section{Discussion}

Nondiscogenic sciatica is rare and unfortunately many clinicians do not have a high index of suspicion. Some of the causes of nondiscogenic sciatica syndrome are not due to degenerative disease but are of oncological pathology and may require urgent investigation or intervention. A significant problem is delay in presentation and delay in diagnosis. Some of the causes of nondiscogenic sciatica syndrome can be difficult to detect in the routine physical examination and even with the standard MRI and CT lumbar spine. Special positions or special projection are often required. CT scans and MRI are both very useful in detecting lesions related to nondiscogenic sciatica syndrome.

MRI demonstrate both the location and the severity of injury. T2W images are useful 
at detecting pathology and may shows high signal intensity of nerve fibers, increased nerve dimension, deformation of nerve or total loss of nerve integrity. MRI neurography is very useful in detecting pathology of the sciatic nerve, throughout its course ${ }^{5}$. Visualization of the lumbosacral plexus in its entirety is difficult with the conventional twodimensional MRI sequence owing to its oblique orientation as reported by Ailianou et al. $^{6}$ they utilized threedimensional Short tau inversion sequences and different flip angles with contrast to detect lesion in the lumbosacral plexus. New modalities also exist such as diffusion tensor imaging (DTI) which help in thorough exploration of even fine anatomical structures. MRI has the ability to reveal high levels of anatomical details of lumbar spine depicting neural structures and soft tissue allowing the diagnosis of intraspinal lesions. Detection of extra spinal causes of sciatica is much more difficult than detection of intra spinal ones and require a high degree of suspicion.

Both the gluteal region and the hip joint regions are common sites for the rare causes of nondiscogenic sciatica syndrome. Yurtseven et al described vascular lesion in the gluteal region causing compression of the sciatic nerve by pseudo aneurysm ${ }^{7}$ Proschek et al. ${ }^{8}$ published a case of posttraumatic false aneurysm of the superior gluteal artery with compression of sciatic nerve leading to nondiscogenic sciatica syndrome. The patient presented with rapidly increasing pain and swelling of the gluteal region, a fall in haemoglobin as well as weak dorsiflexion and eversion of the foot. There was traumatic rupture of the superior gluteal artery following fracture of the pelvis causing compartment syndrome of the buttock ${ }^{9}$. Another case report shows a 90 years old man with atheromatous multiple aneurysm disease developed a rare lesion of the gluteal artery provoked by injury ${ }^{10}$ Even low injury to the gluteal region may produce acute gluteal compartment syndrome which is related to gluteal artery rupture ${ }^{11}$ Inferior gluteal artery injury may also produce gluteal compartment syndrome with sciatic nerve injury following trauma 12. Another rare cause reported was an iatrogenic injury following bone biopsy leading to pseudoaneurysm of the gluteal artery. The patient presented with painful pelvic symptoms, foot drop and sciatica recorded by Phillip et al. ${ }^{13}$. Venous varix in the gluteal region, leading to compression of the sciatic nerve was published by Mark et al. ${ }^{14}$. For the vascular causes of nondiscogenic sciatica syndrome in the gluteal region, a high index of clinical awareness followed by MRI of the gluteal region is advised. Vascular pathology may be an emergency requiring surgical treatment. This report highlights the need for increased index of suspicion particularly when there is a lack of correlation between radiology and the clinical findings, especially if MRI lumbar spine is negative in a patient with cardinal features of sciatica. Special precaution are required for the unconscious patient with multiple injuries because a hidden gluteal injury may missed. Isiklar Z.U. et al. ${ }^{15}$ reported a case of painful neuropathy secondary to intrapelvic migration of acetabular cup, which was diagnosed by plain $\mathrm{X}$ ray of the hip joint. The muscles in the gluteal region can also produce nondiscogenic sciatica syndrome, by compressing the sciatic nerve. Piriformis syndrome is the most common muscular compression to produce nondiscogenic sciatica syndrome. Shane et al confirmed the effects of piriformis muscle in producing nondiscogenic sciatica syndrome ${ }^{16}$. Piriformis syndrome should be high on the differential list when the gluteal region is clinically related to nondiscogenic sciatica syndrome. Gemellus inferior was reported to produce nondiscogenic sciatica syndrome but this is very rare with a paucity of documentation ${ }^{17}$, where in one case report, the muscle was found to be hyperemic and 
tightly compressing the sciatic nerve. Another unusual muscular cause of nondiscogenic sciatica syndrome was confirmed due to the dynamic motion of obturator internus (Murata et al.) ${ }^{18}$ and rupture of quadratus femoris muscle after trauma has also been recognized ${ }^{19}$. In paediatric practice, pyomyositis of the piriformis muscle was discovered as a cause of sciatica ${ }^{20}$. Osteoarthritis of the hip is very common condition in patients older than 65 years, with prevalence of $5 \%$ to $10 \%$ of the population ${ }^{21}$. The sciatic nerve is located in closed proximity to the hip joint and pathology of the hip may involve the sciatic nerve leading to nondiscogenic sciatica syndrome. In the senior author's experience, dual pathology between hip and spine is common where degeneration can occur in both regions.

Another simulator of sciatica is the avascular necrosis the femoral head particularly in the initial stages of the disease. There are many causes of avascular necrosis and MRI is very useful in detecting the necrosis of the femoral head but MRI may looks normal or inconclusive in the initial stages of the disease. Great trochanter bursitis is a cause of pain that may mimics lumbar radiculopathy as per Tortaloni et al. ${ }^{22}$ who confirmed the diagnosis of trochanteric bursitis in $20.2 \%$ of patients who presented to the spine clinic for a presumed lumbar spine pathology with women affected twice as much as men.

Variation in the level of bifurcation and the relation of the sciatic nerve to gluteal muscle, is not rare but it has definite surgical importance. Recognition of this variation in the level of bifurcation will help in the early diagnosis and the treatment of sciatic nerve entrapment or neuropathies and simultaneously reducing the associated morbidities. Bone tumor located in the upper third of the femur may be present with the clinical feature simulating sciatica syndrome ${ }^{23}$. The sacroiliac joint which the senior author refers to as the "hidden and forgotten joint" is a Common site for tuberculosis which may present with clinical feature simulating sciatica ${ }^{24}$. Typhoid spine may simulate sciatica, though it is very rare in European countries. It is endemic in Iraq because of increased risk factors such as lack of raw vegetables, lack of education, untreated water, contact with other diseased patients, eating street food and overcrowding $^{25}$. Brucellosis is another infectious disease affecting the spine, common in the South of Iraq often related to drinking unpasteurized milk. It is a disabling disease and a high index of suspicion is necessary for the diagnosis since there are no pathognomic signs or symptoms. The diagnosis is based on culture of brucella bacilli ${ }^{26}$. Painful diabetic neuropathy, may simulate radiculopathy on clinical background because they present with burning, excruciating, stabbing or even intractable type of pain, in addition to tingling or numbness ${ }^{27}$. Cancer can produce a variety of effects on the nervous system either by direct compression or invasion, or remotely by some metabolic, toxic, viral or immunological affect. Bronchogenic carcinoma is the commonest causes of neuropathy that may appear even several months before the clinical recognition of bronchogenic carcinoma ${ }^{28}$. It is a form of paraneoplastic peripheral neuropathy. The authors confirmed the diagnosis of secondaries in the sciatic nerve from bronchogenic carcinoma. The pelvis is a common site for Ewing sarcoma. Despite advances in adjuvant therapy, Ewing sarcoma of the pelvis remains an anatomic site with a poor prognosis and in the early stage it may cause nondiscogenic sciatica 29. Retroperitoneal sarcoma is a rare tumor, male presents with the Cardinal clinical feature of sciatica, because of its effect on the roots of the sciatic nerve. The most common types of retroperitoneal sarcoma in adult vary from study to study with the most frequently encountered cell types being liposarcoma ${ }^{30}$. Solitary bone plasmacytoma can be challenging to 
diagnose and can often missed; pelvic involvement by these tumors has been rarely described in literature ${ }^{31}$, the location of this pelvic tumor nearby to the course of sciatic nerve may produce sciatica like syndrome. Schwannoma are benign encapsulated tumors derived from the myelin sheath of nerve, originating from Schwann cells and are the most common type of peripheral nerve sheath tumors. They are slow growing and eccentric to the nerve fibers. Schwannoma often do not interfere with nerve function and MRI is the modality of choice to identify them, but it should be noted that it cannot distinguish between malignant and benign tumors $^{32}$. Pain arising from peripheral nerves shoot up and down along the sciatic nerve, giving a clue for the diagnosis of sciatica. Foraminal Schwannoma accounts for 1-5\% of all spinal Schwannoma, it is a rare cause of sciatica and may raise confusion in diagnosis with late discovery 33. Maribel et al reported on malignant peripheral nerve tumors. They are very rare and are frequently localized in the buttock, thigh, arm or paraspinal region ${ }^{34}$. They described a woman presenting with sciatic pain, foot drop with history of lumber disc herniation, which was to later diagnosed to be caused by malignant tumor of the sciatic nerve. Sciatica neuropathy was recorded as the first sign of metastasizing prostate cancer ${ }^{35}$. Intraneural secondaries in the sciatic nerve was recorded from renal cell carcinoma ${ }^{36}$, from advanced urinary bladder carcinoma ${ }^{37}$, and from gastric carcinoma ${ }^{38}$, but the authors could not find any reports of sciatic nerve secondaries from bronchogenic carcinoma in the English literature. Spinal metastases can involve the bone, epidural space, leptomeninges and spinal cord. The spine is the third most common site for metastatic disease, and approximately $60 \%-70 \%$ patients with systemic cancer will have spinal metastasis $^{39}$. Lumbar synovial cysts are cystic dilatations of synovial sheaths that directly emanate from facet joints and protrude in the spinal canal ${ }^{40}$. The cyst compresses the cauda equina and roots leading to nondiscogenic sciatica syndrome and can be diagnosed easily by MRI. Leiomyosarcomas of the paraspinal muscles are variably aggressive tumors of smooth muscle origin. They constitute $0.7 \%$ of all malignancies and arise from sites such as somatic soft tissue of the extremity and trunk. They simulate neurogenic tumors by compressing lumbar roots $^{41}$. Epidural varices are a very rare cause of nondiscogenic sciatica syndrome and gives rise to space occupying lesion resembling a sequestrated disc with clinical feature of sciatica ${ }^{42}$. Intradural spinal tumors include a wide range of histopathological subtype arising from spinal cord, spine nerves and meninges, with the commonest being meningiomas 43 usually in the dorsal region. The best treatment for the benign lesion is excision, though not always easy. Clinically they may present with ideal clinical feature of sciatica. Finally, the diagnosis of nondiscogenic sciatica syndrome remains challenging and delay in the diagnosis remains expected unless there is high index of clinical suspicion. Hopefully the new diagnostic abilities of F18-FDG PetlMRI for neoplasia, which lead to significant uptake in lesion not only in the impinged spinal roots but also with nonspinal cause of pain such as facet joint degeneration, pars defect, presumed scar or neuroma and it may reveal various etiology but so far the number of patients studied is still small ${ }^{44}$.

\section{Conclusion}

- Proper physical examination of the body as whole with emphasis on abdominal, P.R, P.V, and palpation of the sciatic nerve pathway is required in any case of sciatica.

- Thorough investigations, with MRI, and CT scan. 
- A negative physical findings and imaging studies does not exclude a disease in in its initial stage so follow up for the undiagnosed cases is required.

- Rare disease usually hidden under the umbrella of common disease, so clinical awareness is mandatory.
- Poor response to the treatment of discogenic sciatica should raise the suspicion for the nondiscogenic causes.

- There must be a limit for the Conservative treatment of sciatica.

\section{References}

1. Dudeney S, O'Farrell D, Bouchier-Hayes D, J Byrne. Extraspinal causes of sciatica: a case report. Spine 1998; 23:494-496.

2. Swezey R.L. Overdiagnosed Sciatica and Stenosis, Underdiagnosed Hip Arthritis. Orthopedics. 2003; 26(2): 173-174.

3. Fernando Guedes, Rosana Siqueira Brown, Francisco José Lourenço. Nondiscogenic Sciatica: What Clinical Examination and Imaging Can Tell Us? World Neurosurg, 2020; 134: 1053-1061.

4. Bennett Douglas Grimm, Brian Joseph Blessinger, Bruce Vaiden Darden, Craig D Brigham, Jeffrey S Kneisl, Eric B Laxer. Mimickers of Lumbar Radiculopathy. J Am Acad Orthop Surg. 2015; 23(1):7-17.

5. Aaron M Lewis, Robert Layzer, J W Engstrom, Nicholas M Barbaro, Cynthia T Chin. Magnetic Resonance Neurography in Extraspinal Sciatica. Arch Neurol. 2006; 63(10):1469-1472.

6. A Ailianou, A Fitsiori, A Syrogiannopoulou, et al. Review of the Principal Extra Spinal Pathologies Causing Sciatica and New MRI Approaches. Br J Radiol 2012; 85(1014):672-681.

7. Taşkin Yurtseven, Mehmet Zileli, Ege N Tavmergen Göker, Erol Tavmergen, Cüneyt Hoşcoşkun, Mustafa Parildar. Gluteal Artery Pseudoaneurysm, a Rare Cause of Sciatic Pain, Case Report and Literature Review. Journal of Spinal Disorders Techniques 2002; 15(4): 330-333.

8. R Proschek, J V Fowles, L Bruneau. A Case of Post-Traumatic False Aneurysm of the Superior Gluteal Artery with Compression of the Sciatic Nerve. Can J Surg 1983; 26(6):554-555.

9. R J Brumback. Traumatic Rupture of the Superior Gluteal Artery, Without Fracture of the Pelvis, Causing Compartment Syndrome of the Buttock. A Case Report. J Bone Joint Surg Am 1990; 72(1):134-137.

10. Y Glock, Z De Jong, D Roux, E Trocard, G Legrand, S Denat. Aneurysm of the gluteal artery. J Chir (Paris) 1984; 121(11):659-662.

11. Aubrey Smith, Vivek Chitre, Hersh Deo. Acute gluteal compartment syndrome: superior gluteal artery rupture following a low energy injury. BMJ Case Rep. 2012; Dec 17: bcr2012007710.

12. Brett Rocos and Anthony Ward. Gluteal compartment syndrome with sciatic nerve palsy caused by traumatic rupture of the inferior gluteal artery: a successful surgical treatment. BMJ Case Rep. 2017; Jan 25: bcr2016216709.

13. Phillip S. Ge, Gladys Ng, Brandon M. Ishaque, Hugh Gelabert, Christian de Virgilio. Iatrogenic Pseudoaneurysm of the Superior Gluteal Artery Presenting as Pelvic Mass with Foot Drop and Sciatica: Case Report and Review of Literature. SAGE Journal 2009; 44 (1): 64-68.

14. Mark A Pacult, Fraser C Henderson Jr, Mathew D Wooster, Abhay K Varma. Sciatica Caused by Venous Varix Compression of the Sciatic Nerve. World Neurosurgery 2018; 117: 242-245.

15. Z U Isiklar, R W Lindsey, H S Tullos. Sciatic Neuropathy Secondary to Intrapelvic Migration of an Acetabular Cup. A Case Report. J Bone Joint Surg Am 1997; 79(9):1395-1397.

16. Shane P Cass. Piriformis Syndrome: A Cause of Nondiscogenic Sciatica. Curr Sports Med Rep 2015; 14(1):41-44.

17. Arzu Atıcı, Duygu Geler Külcü, Pınar Akpınar, Deniz Akay Urgun. A rare cause of non-discogenic sciatica; musculus gemellus inferior: A case report. Turk J Phys Med Rehabil. 2017; 63(4): 355-356.

18. Yasuaki Murata, Satoshi Ogata, Yoshikazu Ikeda, An Unusual Cause of Sciatic Pain as a Result of the Dynamic Motion of the Obturator Internus Muscle. Spine J 2009; 9(6): 16-18.

19. Artan Bano, Apostolos Karantanas, Dritan Pasku, George Datseris, George Tzanakakis, Pavlos Katonis. Persistent sciatica induced by quadratus femoris muscle tear and treated by surgical decompression: a case report. J Med Case Reports. 2010; 4: 236.

20. Taihei Toda, Masao Koda, Tomoyuki Rokkaku, et al. Sciatica Caused by Pyomyositis of the Piriformis Muscle in a Pediatric Patient. Orthopedics. 2013; 36(2):257-259.

21. Simon Dagenais, Shawn Garbedian, Eugene K. Wai. Systematic Review of the Prevalence of Radiographic Primary Hip Osteoarthritis. Clin Orthop Relat Res. 2009; 467(3): 623-637. 
22. P Justin Tortolani, John J Carbone, Louis G Quartararo. Greater Trochanteric Pain Syndrome in Patients Referred to Orthopedic Spine Specialists. Spine J Jul-Aug 2002; 2(4):251-254.

23. T Nishikawa, T Iguchi, H Honda. Primary Bone Tumors of the Femur Presenting with Spinal Symptoms: A Report of Two Cases and Review of the Literature. J Spinal Disord. 2000; 13(4):360-364.

24. W S Chen. Chronic Sciatica Caused by Tuberculous Sacroiliitis. A Case Report. Spine 1995 May 15; 20(10):1194-1196.

25. Bhutta, Zulfiqar. Typhoid Fever: Current Concepts. Infectious Diseases in Clinical Practice: September 2006; 14(5): 266-272.

26. I H Tekkök, M Berker, O E Ozcan, T Ozgen, E Akalin. Brucellosis of the Spine. Neurosurgery 1993; 33(5): 838-844.

27. Sharonjeet Kaur, Promila Pandhi and Pinaki Dutta. Painful diabetic neuropathy: an update. Ann Neurosci. 2011; 18(4): 168-175.

28. P J Dyck, A Bailey, J Olszewski. Carcinomatous Neuromyopathy; a Case of Sensory Neuropathy and Myopathy with Onset Three and One-Half Years before Clinical Recognition of the Bronchogenic Carcinoma. Can Med Assoc J 1958 Dec 1; 79(11):913-916.

29. Mayil Vahanan Natarajan, M Mohamed Sameer, Jagdish Chandra Bose, Kunal Dheep. Surgical management of pelvic Ewing's sarcoma. Indian J Orthop. 2010; 44(4): 397-401.

30. Isaac R Francis, Richard H Cohan, Datla G K Varma, Vernon K Sondak. Retroperitoneal sarcomas. Cancer Imaging. 2005; 5(1): 89-94.

31. Samir Karmali, André Barros, Barbara Rosa, et al. Solitary Bone Plasmacytoma of the Pelvis: A Rare Tumor. Rare Tumors. 2016 Mar 21; 8(1): 6306.

32. Joseph Maalouly, Rami Ayoubi, Hicham Abdel Nour, et al. Posterior Tibial Nerve Schwannoma Mimicking Tarsal Tunnel Syndrome. Global Journals of Medical Research 2019; 19, No 2-H.

33. C. Karekezi, K. Egu, B. O. Djoubairou, M. Boutarbouch, A. El Ouahabi. Unusual cause of nondiscogenic sciatica: Foraminal lumbar root schwannoma. Surg Neurol Int. 2014; 5(Suppl 4): S208-S210.

34. Maribel R. Gomes, Alexandre M.P. Sousa, Roberto J.A. Couto, Marco M B Oliveira, João L M Moura, Carlos A Vilela. Malignant triton tumor, a rare cause of sciatic pain and foot drop. Rev Bras Ortop. 2017; 52(4): 496-500.

35. Jakob Møller Hansen, Zoreh Rasti, Torben Smith, Lisbeth Hjorth Lassen. Sciatic neuropathy as first sign of metastasising prostate cancer. BMJ Case Reports 2010(oct08 2). DOI: 10.1136/ bcr.12.2009.2529.. 36. Varin S, Faure A, Bouc P, Maugars Y, Berthelot JM. Endoneural metastasis of the sciatic nerve disclosing the relapse of a renal carcinoma, four years after its surgical treatment. Joint Bone Spine, 04 Oct 2006; 73(6):760-762.

37. E Nagao, A Nishie, K Yoshimitsu, et al. Gluteal Muscular and Sciatic Nerve Metastases in Advanced Urinary Bladder Carcinoma: Case Report. Abdom Imaging Sep-Oct 2004; 29(5):619-622.

38. Jiro Ichikawa, Seiichi Matsumoto, Takashi Shimoji, et al. Intraneural metastasis of gastric carcinoma leads to sciatic nerve palsy. BMC Cancer. 2012; 12: 313.

39. Lubdha M. Shah and Karen L. Salzman.Imaging of Spinal Metastatic Disease. Int J Surg Oncol. 2011; 769753.

40. Nancy E Epstein, Jamie Baisden. The Diagnosis and Management of Synovial Cysts: Efficacy of Surgery versus Cyst Aspiration. Surg Neurol Int 2012; 3(Suppl 3): 157-166.

41. Duygu Yazgan Aksoy, M Kadri Altundag, Mine Durusu, et al. Thoracic Paravertebral Leiomyosarcoma: Rare but It Does Occur. Spine (Phila Pa 1976) 2002 Jun 15; 27(12): 301-303.

42. Farzad Omidi-Kashani, Ebrahim Ghayem Hasankhani, Mahdi Fathi. Primary Epidural Varicosis as a Rare Cause of Sciatica: A Case Report. Iran J Med Sci. 2015; 40(6): 541-543.

43. Muhammad M Abd-El-Barr, Kevin T Huang, Ziev B Moses, J Bryan Iorgulescu, John H Chi. Recent advances in intradural spinal tumors. Neuro Oncol. 2018; 20(6): 729-742.

44. Peter W. Cipriano, Daehyun Yoon, Harsh Gandhi, et al. 18F-FDG PET/MRI in Chronic Sciatica: Early Results Revealing Spinal and Nonspinal Abnormalities. J Nucl Med 2018; 59(6):967-972. 